VOL. 63 (2001) [341-344]

\title{
POWER GRAPHS AND SEMIGROUPS OF MATRICES
}

\author{
A.V. Kelarev, S.J. Quinn and R. Smolíková
}

\begin{abstract}
Matrices provide essential tools in many branches of mathematics, and matrix semigroups have applications in various areas. In this paper we give a complete description of all infinite matrix semigroups satisfying a certain combinatorial property defined in terms of power graphs.
\end{abstract}

Research on combinatorial properties of words in groups originates from the following well-known theorem due to Bernhard Neumann [12], which was obtained as an answer to a question of Paul Erdős: a group is centre-by-finite if and only if every infinite sequence contains a pair of elements that commute. Combinatorial properties of groups and semigroups with all infinite subsets containing certain special elements have been considered by Bell, Blyth, Curzio, de Luca, Gillam, Hall, Higgins, Justin, Longobardi, Maj, Okniński, Piochi, Pirillo, Restivo, Reutenauer, Rhemtulla, Robinson, Sapir, Shumyatsky, Simon, Varricchio and other authors, and a survey of this direction was given by the first author in $[7]$ (see also $[2,3,6,11]$ ).

The following combinatorial property was introduced in [9] using power graphs. The power graph Pow(S) of a semigroup $S$ has all elements of $S$ as vertices, and it has edges $(u, v)$ for all $u, v \in S$ such that $u \neq v$ and $v$ is a power of $u$. Let $D$ be a directed graph. We say that an infinite semigroup $S$ is power $D$-saturated if and only if, for every infinite subset $T$ of $S$, the power graph of $S$ has a subgraph isomorphic to $D$ with all vertices in $T$. In this paper we describe all pairs $(D, S)$, where $D$ is a directed graph and $S$ is a matrix semigroup, such that $S$ is power $D$-saturated.

The reader is referred to $[\mathbf{1}, \mathbf{4}, \mathbf{1 4}]$ for standard graph, semigroup and group theoretic terminology, respectively. By the word 'graph' we mean a directed graph without loops or multiple edges. A graph is said to be acyclic if it has no directed cycles.

We refer to $[10,13]$ for preliminaries on fields and matrix semigroups, respectively. For a skew field $K$, the set of all $n \times n$ matrices with entries in $K$ is denoted by $M_{n}(K)$. A matrix is said to be monomial if every row and column contains at most one nonzero entry. If $G$ is a group, then the set of all $n \times n$ monomial matrices over $G^{0}=G \cup\{0\}$ forms a semigroup denoted by $M_{n}(G)$ (see [5]). Thus a matrix semigroup is a subsemigroup of $M_{n}(K)$ or $M_{n}(G)$, for some $n, K$ and $G$.

Received 28th August, 2000

Copyright Clearance Centre, Inc. Serial-fee code: 0004-9727/01 \$A2.00+0.00. 
Obviously, if $D$ is a null graph, that is, a graph without edges, then all semigroups are power $D$-saturated.

THEOREM 1. Let $D=(V, E)$ be a directed graph with $E \neq \emptyset, K$ a skew field, $G$ a group, and let $S$ be an infinite matrix semigroup in $M_{n}(K)$ or in $M_{n}(G)$. Then $S$ is power $D$-saturated if and only if $D$ is acyclic and all but a finite number of elements of $S$ are contained in the union of a finite number of centre-by-finite torsion groups $H_{i}$, where $i=1, \ldots, k$, such that the centre $C\left(H_{i}\right)$ of each $H_{i}$ has a finite number of primary components, each primary component of $C\left(H_{i}\right)$ is finite or quasicyclic, and the order of $H_{i} / C\left(H_{i}\right)$ is not divisible by $p$ for each quasicyclic $p$-subgroup of $H_{i}$.

We use the following technical lemmas, found in $[5,13]$ (see also $[8]$ ). Let $G L_{j}(K)$ be a maximal group of matrices of rank $j$ over $K$. Put

$$
M_{j}=\left\{a \in M_{n}(K) \mid \operatorname{rank}(a) \leqslant j\right\} .
$$

Lemma 2. [5] Let $G$ be a group. Then $M_{n}(G)$ is an inverse semigroup with the only ideals

$$
\{0\}=M_{0} \subset M_{1} \subset \cdots \subset M_{n}=M_{n}(G),
$$

where $M_{j}=\{s \mid s$ has at most $j$ nonzero entries $\}$. Moreover,

$$
M_{j} / M_{j-1} \cong \mathcal{M}\left(G_{j},\left(\begin{array}{c}
n \\
j
\end{array}\right),\left(\begin{array}{c}
n \\
j
\end{array}\right), \Delta\right)
$$

where $G_{j}$ is an extension of $G^{j}=G \times \cdots \times G$ by the symmetric group $S_{j}$ and $\Delta$ is the identity matrix. All idempotents of $M_{n}(G)$ are diagonal and a power of every element is diagonal.

Lemma 3. [13, Theorem 2.3] The sets

$$
\{0\}=M_{0} \subset M_{1} \subset \cdots \subset M_{n}=M_{n}(K)
$$

are the only ideals of the monoid $M_{n}(K)$. Each Rees factor $M_{j} / M_{j-1}$ is isomorphic to the completely 0-simple semigroup $\mathcal{M}\left(G L_{j}(K), X_{j}, Y_{j}, Q_{j}\right)$, where the matrix $Q_{j}=\left(q_{y x}\right)$ is defined for $x \in X_{j}, y \in Y_{j}$, by $q_{y x}=y x$ if $y x$ is of rank $j$ and 0 otherwise.

Throughout the rest of the paper $M_{n}$ denotes one of the semigroups $M_{n}(K)$ or $M_{n}(G)$. It is easily seen that the sets $M_{j} \backslash M_{j-1}$ in the lemmas above correspond to the $\mathcal{D}$-classes of $M_{n}$. We also need the following result obtained in [9]:

Proposition 4. [9] Let $D=(V, E)$ be a graph with $E \neq \emptyset$ and let $H$ be an infinite group. Then $H$ is power $D$-saturated if and only if $H$ is a centre-by-finite torsion group, the centre $C(H)$ has a finite number of primary components, each primary component of $C(H)$ is finite or quasicyclic, the order of $H / C(H)$ is not divisible by $p$ for each quasicyclic $p$-subgroup of $H$, and $D$ is acyclic. 
ProOF OF THEOREM 1: In the full linear or monomial semigroup $M_{n}$, let us consider the chain of ideals $M_{0}, M_{1}, \ldots, M_{n}$, defined in Lemma 3 and Lemma 2.

The 'only if' part. Suppose that $S$ is power $D$-saturated. Then every infinite subset of $S$ induces a subgraph of Pow(S) isomorphic to $D$.

Consider the set $T$ of all elements of $S$ contained in the $\mathcal{H}$-classes of $M_{n}$ which are not groups. Suppose that $T$ is infinite. By the definition of $T$ and $M_{j}$, we get:

$$
T \subseteq S \subseteq M_{n}=\cup\left\{M_{j} \backslash M_{j-1} \mid 1 \leqslant j \leqslant n .\right\}
$$

So, $T=\bigcup\left\{T \cap\left(M_{j} \backslash M_{j-1}\right) \mid 1 \leqslant j \leqslant n\right\}$, and hence at least one $T \cap\left(M_{j} \backslash M_{j-1}\right)$ must be infinite. Now, if $s \in T \cap\left(M_{j} \backslash M_{j-1}\right)$, then by [4, Theorem 3.3.1], we know that $s^{k} \in M_{j-1}$ for all $k \geqslant 2$. Hence all elements of $T \cap\left(M_{j} \backslash M_{j-1}\right)$ are not adjacent in Pow(S). Given that $D$ has edges, we see that the subgraph induced by $T \cap\left(M_{j} \backslash M_{j-1}\right)$ in Pow (S) does not have a subgraph isomorphic to $D$. This contradicts the power $D$-saturation of $S$, and shows that $T$ is finite.

Suppose that the elements of $S \backslash T$ belong to' infinitely many $\mathcal{H}$-classes of $M_{n}$. The definition of $T$ shows that all these $\mathcal{H}$-classes are groups. By the axiom of choice we can form a subset $Q$ which contains exactly one element of each $\mathcal{H}$-class of $M_{n}$ intersecting $S \backslash T$. Then it follows that $Q$ is infinite and induces a null subgraph in Pow(S). Again, this contradicts power $D$-saturation, and shows that $S \backslash T$ is contained in a finite number of $\mathcal{H}$-classes of $M_{n}$.

Take any $\mathcal{H}$-class $Q$ of $M_{n}$ intersecting $S \backslash T$. Put $R=Q \cap S$. If $R$ has an element $r$ of infinite period, then the vertices $r^{2}, r^{3}, r^{5}, r^{7}, \ldots$ are not adjacent in the power graph of $S$. This contradicts power $D$-saturation again, and shows that all elements in $R$ are periodic. Since $Q$ is a group, we see that $R$ is a group too.

Thus $S \backslash T$ is a union of a finite number of groups. The power $D$-saturation is inherited by subsemigroups, and so all these groups are also power $D$-saturated. Evidently, all but a finite number of elements of $S \backslash T$ are contained in the union of a finite number of infinite groups $H_{i}$, where $i=1, \ldots, k$. Then by Proposition 4 , each $H_{i}$ is a centre-by-finite torsion group such that the centre $C\left(H_{i}\right)$ of $H_{i}$ has a finite number of primary components, each primary component of $C\left(H_{i}\right)$ is finite or quasicyclic and the order of $H_{i} / C\left(H_{i}\right)$ is not divisible by $p$ for each quasicyclic $p$-subgroup of $H_{i}$.

It remains to verify that the graph $D$ is acyclic. Given that $S$ is infinite, it has an infinite subgroup $R$. Hence $R$ contains a quasicyclic subgroup $C_{p}^{\infty}$. Let $r_{1}, r_{2}, \ldots$ be generators of $C_{p}^{\infty}$, such that $r_{1}^{p}=e$ and $r_{i}^{p}=r_{i-1}$. Then it is routine to verify that the set $\left\{r_{1}, r_{2}, \ldots\right\}$ induces a subgraph which is isomorphic to an infinite chain, which is of course acyclic. By the power $D$-saturation of $S$, we see that $D$ embeds in the chain, and so $D$ is acyclic too.

The 'if' part. By Proposition 4, we may assume that all but a finite number of elements of $S$ are contained in the union of a finite number of power $D$-saturated groups. 
Then every infinite subset $T$ of $S$ contains an infinite subset $U$ of $T$ such that $U \subseteq(S \cap R)$, where $R$ is a group. By assumption $S \cap R$ is power $D$-saturated and therefore $D$ embeds in Pow(S).

\section{REFERENCES}

[1] G. Chartland and L. Lesniak, Graphs and digraphs (Chapman \& Hall, London, 1996).

[2] A. de Luca and S. Varricchio, 'Regularity and finiteness conditions', in Handbook of Formal Languages 1, (G. Rosenberg and A. Salomaa, Editors) (Springer-Verlag, Berlin, Heidelberg, New York, 1997), pp. 747-810.

[3] A. de Luca and S. Varricchio, Finiteness and regularity in semigroups and formal languages, Monographs in Theoretical Computer Science (Springer-Verlag, Berlin, Heidelberg, New York, 1998).

[4] J.M. Howie, Fundamentals of semigroup theory (Clarendon Press, Oxford, 1995).

[5] E. Jespers and J. Okniński, 'On a class of Noetherian algebras', Proc. Roy. Soc. Edinburgh Sect. $A 129$ (1999), 1185-1196.

[6] J. Justin and G. Pirillo, 'On some questions and conjectures in combinatorial semigroup theory', Southeast Asian Bull. Math. 18 (1994), 91-104.

[7] A.V. Kelarev, 'Combinatorial properties of sequences in groups and semigroups', in Combinatorics, Complexity and Logic, (D.S. Bridges, C.S. Calude, J. Gibbons, S. Reeves and I.H. Witten, Editors), Springer Ser. Discrete Math. Theor. Comput. Soc. (Springer-Verlag, Singapore, 1997), pp. 289-298.

[8] A. V. Kelarev, 'Radicals of algebras graded by cancellative linear semigroups', Proc. Amer. Math. Soc. 124 (1996), 61-65.

[9] A.V. Kelarev and S.J. Quinn, 'A combinatorial property of power graphs of groups', in Contrib. General Algebra 12 (Heyn, Klagenfurt, 2000), pp. 229-235.

[10] R. Lidl and G. Pilz, Applied abstract algebra (Springer-Verlag, Berlin, Heidelberg, New York, 1998).

[11] M. Lothair, Combinatorics on words (Addison-Wesley, Tokyo, 1982).

[12] B.H. Neumann, 'A problem of Paul Erdös on groups', J. Austral. Math. Soc. 21 (1976), 467-472.

[13] J. Okniński, Semigroups of matrices (World Scientific, Singapore, 1998).

[14] D.J.S. Robinson, A Course in the theory of groups (Springer-Verlag, Berlin, Heidelberg, New-York, 1982).

Department of Mathematics

University of Tasmania

G.P.O. Box 252-37

Hobart Tas. 7001

Australia

e-mail: Andrei.Kelarev@utas.edu.au
Department of Mathematics

University of Tasmania

Launceston Tas 7250

Australia

e-mail: Stephen.Quinn@utas.edu.au

Department of Electrical Engineering

University of Louisville

United States of America

e-mail: r0smol01@agy.spd.louisville.edu 